\section{Prostatakrebs-Patienten geben ihren Ärzten Bestnoten}

\section{Patienten mit Prostatakrebs fühlen sich von ihren Urologen bestens betreut. Dieses positive Urteil belegt die sehr gute onkologische Kom- petenz der Urologen in Deutschland.}

Seit 2008 werden Patienten- und Arztangaben zu Tumorcharakteristika, Lebensqualität, Krankheitserleben und Arzt-Patient-Beziehung erfasst und ausgewertet.

Dem Zwischenbericht der HAROWStudie zufolge haben Patienten eine hervorragende Meinung von ihren

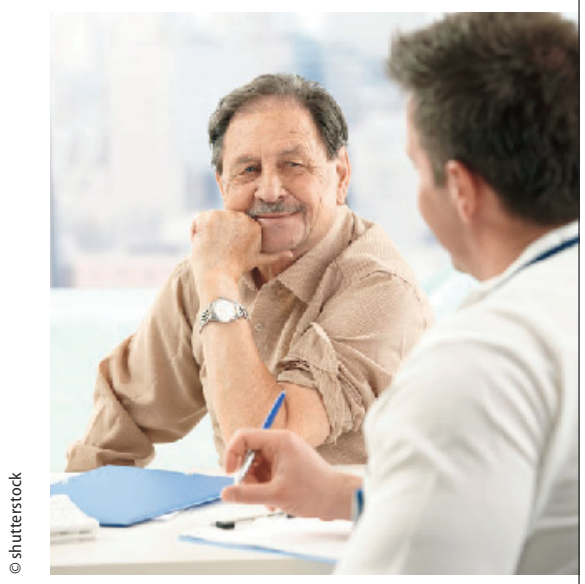

Urologen. Die meisten bewerten die Arzt-Patienten-Interaktionen als sehr gut und nehmen die niedergelassenen Urologen auch als ausgezeichnete Onkologen wahr. Ihnen wird eine hohe psychosoziale Versorgungsqualität und ärztliche Empathie attestiert. Fast $90 \%$ der Patienten haben einen festen Ansprechpartner in der urologischen Praxis. Mehr als $40 \%$ der Urologen sind sogar außerhalb der Sprechzeiten für ihre Patienten da. Diese können sich zudem genügend Zeit für wichtige therapeutische Entscheidungen nehmen und fühlten sich von ihren Urologen über die verschiedenen Therapieoptionen verständlich informiert.

Quelle: Berufsverband der Deutschen Urologen e.V.

Abb. 3 Mögliche Mechanismen der Androgenresistenz (AR: Androgenrezeptor). (Mit freundlicher Genehmigung der Nature Publishing Group, nach [13])

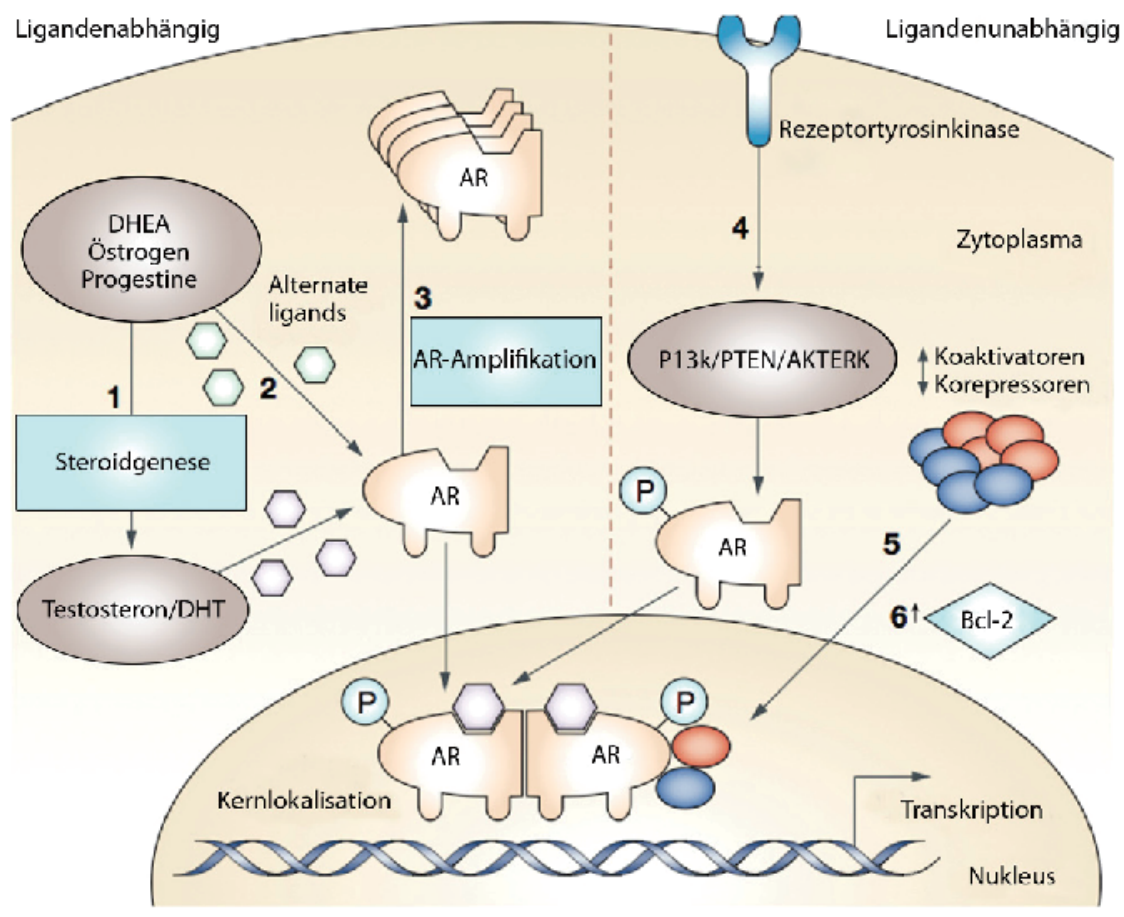

so wurde für die mit Bevacizumab behandelten Patienten ein schlechteres Toxizitätsprofil mit mehr Nebenwirkungen verzeichnet [12].

Nach den vielversprechenden Ergebnissen einer vorausgegangenen Phase-IIStudie zum Vergleich Docetaxel \pm Calcitriol (hochdosiertes Vitamin D) wurde in der sogenannten ASCENT-II-Studie, einer randomisierten Phase-III-Studie, kürzlich ein Überlebensnachteil von Patienten mit der Kombinationsbehandlung Docetaxel und Calcitriol berichtet. Die Studie wurde daraufhin im Herbst 2008 abgebrochen. Nach neuesten Analysen, die auf dem ASCO-Kongress vorgestellt wurden, handelte es sich hier hochwahrscheinlich nicht um Calcitriol-, sondern um PCA-bedingte Todesfälle. Es konnte jedoch nochmals bestätigt werden, dass das 3-wöchige Docetaxel-Schema dem wöchentlichen Schema deutlich überlegen ist.

Ein interessanter Ansatz könnte auch die Inhibition der sog. Endothelin-Achse beim CRPC sein. Aus präklinischen Studien ist bekannt, dass der Enothelin-ARezeptor beim CRPC signifikant erhöht ist. Derzeit wird in einem großen PhaseIII-Studienprogramm die Substanz Zibotentan (ZD4054) sowohl als Monotherapie wie auch als Kombinationstherapie mit Docetaxel getestet. Ergebnisse werden in naher Zukunft erwartet.

In einer weiteren Studie wurde jüngst der Tyrosinkinaseinhibitor Sunitinib als Second-Line-Option nach DocetaxelVersagen getestet, nachdem diese Substanz in einer vorausgehenden Phase-IIStudie bereits interessante Ergebnisse erbracht hatte. Die aktuelle placebokontrollierten Phase-III-Studie wurde allerdings frühzeitig gestoppt, da in einer zweiten Zwischenauswertung für Sunitinib kein Überlebensvorteil, jedoch eine höhere Grad-3/4-Nebenwirkungsrate nachgewiesen wurde.

\section{Neue antihormonelle Ansätze}

Zwei neue Substanzen aus dem Bereich der antihormonellen Therapien werden derzeit in einem großen Studienprogramm beim PCA getestet:

- MDV310o, ein neuartiges Antiandrogen mit insgesamt 3 Wirkungsansätzen, blockiert den Androgenrezeptor 